\title{
Cancer immunotherapy making headway
}

Harnessing the immune system to more effectively fight diseases has long been adopted and recently made great strides in cancer treatment. The promise of more specific, less toxic anti-tumour response through immunotherapy and vaccination compared to conventional therapeutics in cancer piqued the interest of researchers and led to the development of innovative approaches. One approach has been the use of checkpoint inhibitors that eliminate the 'brakes' on the immune system that can prevent immune cells from attacking cancer cells. Another approach is adoptive cell therapy, which involves the use of immune cells that are re-engineered to better recognize cancer cells and attack them. Cancer vaccines have also been adopted and involve the recognition of tumour antigens as 'non-self', ultimately stimulating the immune system to mount an effective anti-tumour response. However, a series of clinical trials have highlighted the need for caution and selectivity in their use due to the risks associated with autoimmunity and off-target toxicity. We asked experts in the field of immunotherapy and vaccine development to offer their opinion on new and existing therapeutics, as well as the challenges and successes in clinical use. In particular, they discuss the use of innovative materials in vaccine development and how they can enhance the potency and safety of vaccines. Moreover, they also discuss the innovative approaches in the use of adoptive cell therapies and dendritic cell vaccines that have been employed to generate anti-tumour immunity by activating tumour-specific lymphocytes. Other innovative approaches such as the generation of an immune-response against multiple tumour antigens by DNA demethylation agents are also discussed as well as the recent clinical trials that highlight the promise of immunotherapy in treating cancer.

Amos Matsiko

Nature Materials.

e-mail:amos.matsiko@nature.com

Published online: 23 May 2018

https://doi.org/10.1038/s41563-018-0091-8

\section{Material aid for vaccines}

Darrell Irvine provides an overview of the recent advances in materials science that have enabled the use of innovative natural and synthetic compounds in vaccine development capable of regulating the potency and safety of new vaccines progressing towards the clinic.

\footnotetext{
ffective vaccines have been a critical part of modern public health since the early twentieth century, and have helped to eradicate smallpox - and will probably eradicate polio as well - while dramatically lowering the global burden of dozens of other infectious diseases. However, efforts are still ongoing to develop fully effective vaccine strategies for many pathogens that establish chronic infections (for example, HIV, malaria, tuberculosis), and emerging/recurrent viruses such as pandemic flu, Ebola and Zika virus present
}

new threats to be addressed. In addition, tolerogenic vaccines are envisioned to deal with immune responses against biologic drugs, autoimmune disorders and transplant rejection. Therapeutically, there is a resurgent interest in vaccines against cancer, especially vaccines that target so-called neoantigens, mutations in tumour proteins that can be safely recognized as non-self by the immune system. New technologies have often had an important role in modern vaccine development, and materials-based approaches to vaccine adjuvants and vaccine delivery are playing a key role in addressing these challenges. Importantly, a number of promising technologies are moving out of the lab and into the clinic to address the outstanding challenges noted above.

The majority of new vaccines in development for infectious diseases are 'subunit' vaccines, which include a well-defined protein or polysaccharide antigen derived from the pathogen that is the target for protective antibodies or, in some cases, $\mathrm{T}$ cell responses. 
These antigens must be combined with adjuvants, materials that stimulate the immune system to promote recognition of the antigen and mount an effective immune response. Over the past 20 years, a broad set of innate immune receptors have been defined that allow immune cells to sense specific molecular signatures of microbes. These include, for example, Toll-like receptors (TLRs) that recognize bacterial lipopolysaccharides, viral RNA and DNA, and fungal polysaccharides. Ligands for TLRs have long been considered attractive vaccine adjuvant candidates, because they are single molecular agents acting on defined pathways. However, the potency of these compounds in triggering inflammatory pathways is a double-edged sword, as overstimulation at the injection site or leakage of these compounds into the systemic circulation can lead to side effects such as flu-like symptoms that are unacceptable for prophylactic vaccines. Recently several materials chemistry innovations have been demonstrated that illustrate clever ways to harness these promising compounds. Novartis developed a series of small molecule immune potentiators (SMIPs), a library of compounds that precisely stimulate chosen TLRs. As small molecules, many of these agents had unfavourable pharmacokinetics (for example, rapid dissemination into the blood following injection). To overcome this limitation, these immunomodulators were conjugated with phosphate linkers that undergo a high-affinity ligand exchange reaction with the surface of one of the most common licensed adjuvants, aluminium hydroxide (alum). By binding to alum, the compounds were prevented from leaking into the blood and were instead ferried primarily to lymph nodes by innate immune cells, leading to inflammation focused only in the draining lymph node - exactly where it is needed in a vaccine ${ }^{1}$. This approach has shown excellent results in non-human primates, an important animal model for human vaccine development. Novartis sold their vaccine portfolio to GSK, one of the world's other major vaccine manufacturers, and GSK is continuing to move these novel adjuvants forward toward clinical testing. A second promising approach is to conjugate TLR agonists to polymers that self-assemble into particulates of an appropriate size to carry them to lymph nodes from an injection site. This strategy was shown to greatly enhance the potency and safety of a small molecule TLR adjuvant in mouse models ${ }^{2}$, and is now being moved toward clinical testing by Avidea Technologies.
In cancer, two recent clinical trials reported results from the first personalized cancer vaccines targeting neoantigens, patient-specific cancer mutations. These and many encouraging preclinical studies have motivated new interest in strategies to develop therapeutic cancer vaccines, and several different materials-based technologies are in late preclinical/early clinical development with the specific goal of promoting $\mathrm{T}$ cell-based immunity required for cancer vaccines. The simplest form of neoantigen vaccines is based on the injection of synthetic peptide antigens, but these generally elicit weak immune responses. Several companies are developing new ways to enhance peptide vaccines: EVOQ Therapeutics is employing lipid nanodiscs that mimic lipoproteins for coformulation of peptides and adjuvant compounds ${ }^{3}$. Another start-up company, Vedantra Pharmaceuticals, is developing approaches to enhance peptide vaccines through polymeric conjugates that target antigens or adjuvant compounds to lymph nodes via reversible binding to albumin ${ }^{4}$. Recently, Novartis announced a partnership with the Wyss Institute and Harvard University to develop implantable and injectable scaffolds that carry antigens and immunostimulatory compounds to prime anti-tumor immunity, translating years of promising preclinical work from the laboratory of David Mooney at Harvard ${ }^{5}$. Thus, a diverse array of new technologies are being moved toward clinical testing for vaccine-based cancer immunotherapy.

Beyond peptide and protein-based vaccines, a major area of research is in the development of materials to deliver engineered RNA expressing antigens for therapeutic or prophylactic immunization. Here again there are many recent translational advances; too many to comprehensively mention here. BioNTech in Europe has carried out a pilot clinical trial intravenously administering mRNA vaccines formulated in lipid nanoparticles in cancer patients with the goal of targeting systemic dendritic cells ${ }^{6}$. Valera, a spin-off of Moderna focused on infectious diseases, has reported promising immunogenicity of mRNA-encoded influenza vaccines delivered by lipid nanoparticles in a phase I clinical trial ${ }^{7}$. CureVac has also developed mRNA vaccines, formulated as nanoparticles in complex with the cationic protein protamine, and recently reported induction of neutralizing antibody responses to a rabies vaccine in humans ${ }^{8}$. Novartis developed cationic nanoemulsions to deliver self-replicating RNAs for vaccination, and reported impressive immunogenicity in non-human primates at modest RNA doses ${ }^{9}$; GSK continues to develop this technology for human translation. Further enhancements in these nucleic acid platforms will be driven in part by improving the delivery materials used in these vaccines.

A final area of exciting recent advances is the development of tolerogenic vaccines that block unwanted immune responses. Selecta Biosciences has developed an approach employing biodegradable polylactide/poly(lactideco-glycolide) nanoparticles carrying the immunosuppressive agent rapamycin, which can be co-administered with an antigen to promote tolerance to the latter molecule ${ }^{10}$. The company is currently in phase two clinical trials for a treatment to induce tolerance to recombinant uricase, an enzyme that is used to treat patients with gout but that is seen by the immune system as a foreign molecule. If successful, this system could provide a generic blueprint for promoting antigen-specific tolerance.

Altogether these examples illustrate much promise in the use of new materials and materials chemistries to control the biodistribution, mechanisms of action, safety and potency of new vaccines. Clinical success of these technologies will pave the way for further development of such innovative approaches and provide important new information about the impact of materials-based vaccine strategies in humans.

\section{Darrell Irvine $e^{1,2,3}$ \\ ${ }^{1}$ Department of Biological Engineering, Department of Materials Science and Engineering, and Koch Institute for Integrative Cancer Research, MIT, Cambridge, MA, USA. ${ }^{2}$ Ragon Institute of MGH, MIT and Harvard, Cambridge, MA, USA. ${ }^{3}$ Howard Hughes Medical Institute, Chevy Chase, MD, USA. e-mail:djirvine@mit.edu}

\section{Published online: 23 May 2018}

https://doi.org/10.1038/s41563-018-0089-2

References

1. Wu, T. Y.-H. et al. Sci. Transl. Med 6, 263 ral60 (2014).

2. Lynn, G. M. et al. Nat. Biotechnol. 33, 1201-1210 (2015).

3. Kuai, R., Ochyl, L. J., Bahjat, K. S., Schwendeman, A. \& Moon, J. J. Nat. Mater. 16, 489-496 (2017)

4. Liu, H. et al. Nature 507, 519-252 (2014).

5. Ali, O. A., Huebsch, N., Cao, L., Dranoff, G. \& Mooney, D. J. Nat. Mater. 8, 151-158 (2009).

6. Kranz, L. M. et al. Nature 534, 396-401 (2016).

7. Bahl, K. et al. Mol. Ther. 25, 1316-1327 (2017).

8. Alberer, M. et al. Lancet 390, 1511-1520 (2017).

9. Brito, L. A. et al. Mol. Ther. 22, 2118-2129 (2014).

10. Maldonado, R. A. et al. Proc. Natl Acad. Sci. USA 112, E156-E165 (2015). 